\title{
DEVELOPMENT OF A LOWER LIMB STROKE REHABILITATION MACHINE
}

\author{
Khuan Foo Law ${ }^{1}$, Dalbir Singh ${ }^{1}$ and Zaidi Mohd Ripin ${ }^{1}$ \\ ${ }^{1}$ TheVibrationLab, \\ School of Mechanical Engineering, \\ Universiti Sains Malaysia, \\ 14300 Nibong Tebal, SPS, \\ Pulau Pinang, Malaysia. \\ *Email: mezaidi@usm.my \\ Phone: +6045996368; Fax: +6045996912
}

\begin{abstract}
This paper explains the development of a lower limb stroke rehabilitation machine designed for subacute stroke patients. The system is capable of measuring the muscle force and providing goal-oriented feedback in real-time and running in two different rehabilitation modes. The mean value of engagement metric of healthy subjects using this machine with feedback was $24.53 \%$ higher than without feedback. This proved that feedback can help the patients to be fully engaged during the rehab session and this can be useful in strengthening the neuromotor pathways. The brain recovery based on the motor cortex correlation quantification algorithm based on the electroencephalography (EEG) signals which is validated against the established technique based on the functional magnetic resonance imaging (fMRI). From the results, the resting-state EEG beta coherence of healthy subjects was found to be $0.474 \pm 0.06$, whereas the average fMRI functional connectivity between left and right primary motor areas of healthy subjects was $0.537 \pm 0.08$. The percentage difference was only $11.7 \%$. clinical trial will be carried out to further measure the efficacy of the rehabilitation treatment using this system.
\end{abstract}

Keywords: electroencephalography, functional connectivity, functional magnetic resonance imaging, lower limb, motor cortex, recovery, rehabilitation, stroke, subacute and visual feedback.

\section{INTRODUCTION}

According to the World Heart Federation, 15 million people worldwide suffer from stroke and nearly five million are left permanently disabled with paralysis and weaknesses every year [1]. For instance, approximately 50,000 Canadians and 780,000 Americans suffer a stroke each year, One-sided paralysis is a common effect of stroke which diminishes the strength and control of the lower limb [2,3]. About $80 \%$ of patients with stroke experience motor weakness/hemiparesis [4].

The functional recovery and long-term health status of the stroke patients are more affected by rehabilitation during the subacute stage [5]. Generally, the recovery from stroke occurs within 3 months after the onset of stroke. To be more specific, it is proven that the neurological and functional recovery of stroke patients occurs at the 
higher rate during subacute stage [6]. Rehabilitation at the sub-acute stage is difficult due to the fact that the patients are generally not strong enough to carry out exercise of the lower limb. In this project, a small, lightweight and portable rehabilitation machine is developed specifically for the stroke patient to carry out rehabilitation exercise which still on the bed. Commonly, stroke rehabilitation guidelines recommend the intensive and repetitive practice of functional tasks after stroke [7]. Moving the limb in a manner that self-generated effort cannot achieve, assistive exercise provides novel somatosensory stimulation that helps induce brain plasticity [8]. Studies [9-10] suggest that providing too much assistance may have negative consequences for learning. Example strategies to encourage participant effort and self-initiated movements by implementing the active resistive exercise, triggering assistance only when participants achieve a force or velocity threshold, making robot compliant [11]. The brain is able to rebuild the damaged neuron paths through the conscious control of the limb's motion [12] and this can be achieved through movement re-education [13, 14, 15]. In terms of quantification of stroke recovery, it is commonly indicated by Fugl Meyer Assessment (FMA) score. It is a stroke-specific, performance-based impairment index and is designed to assess motor functioning, coordination, balance, sensation and joint functioning in patients with post-stroke hemiplegia [16]. However, its scoring is based on direct observation of performance and subjective evaluation and broad based. Hence, a more specific and direct measurement is needed and in this project, the push force of the lower limb during flexion-extension movement can be obtained.

In order to tackle all the stated problems related to the rehabilitation of the lower limbs for stroke patients, three main objectives have been highlighted as below:

1. To design a lower limb rehabilitation machine which can monitor muscle strength recovery.

2. To investigate the engagement of stroke patients in rehabilitation exercises.

\section{METHODOLOGY}

\section{Design of the lower limb rehabilitation machine}

The view of the machine with all the major components is as shown in Fig. 1 below. In this figure, the machine is shown with load cells which are used to measure the force applied by the lower limb of stroke patients throughout the rehabilitation exercises, as an indication of the lower limb motor performance. Meanwhile, ultrasonic sensors are used for the measurement of the range of motion, and eventually to provide real-time visual feedback on the screen. 


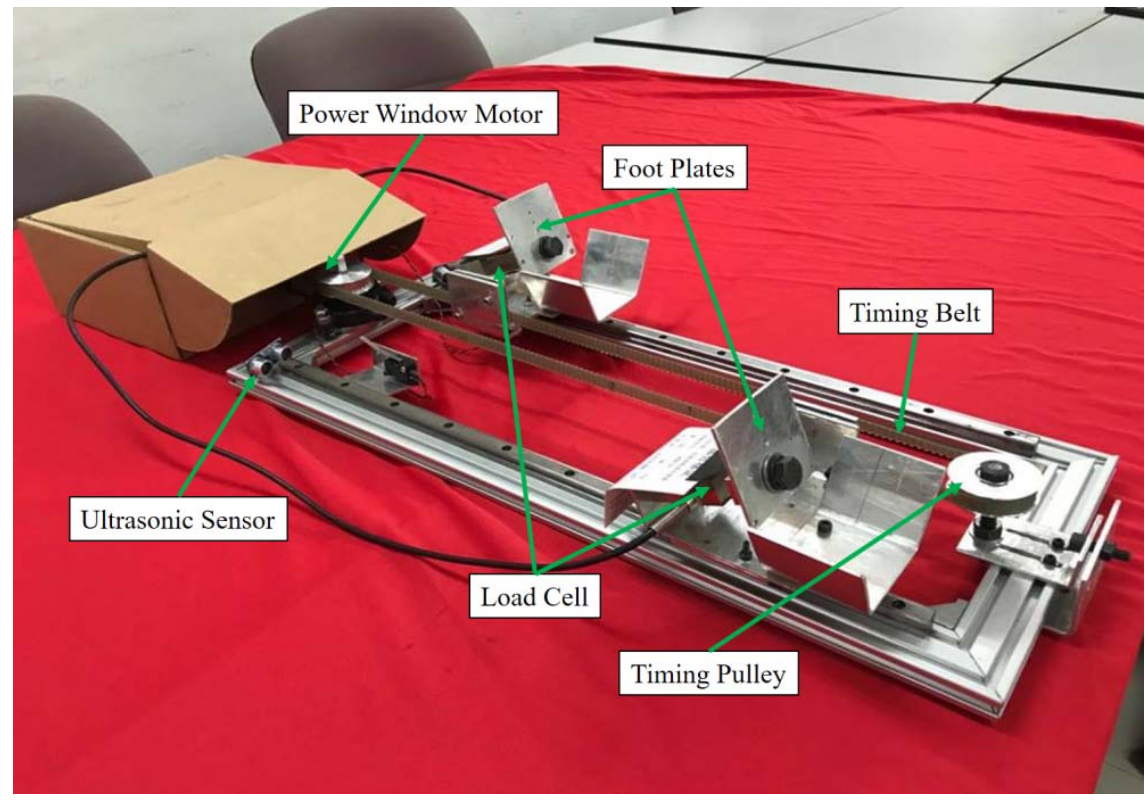

Figure 1. Overview of the stroke rehabilitation machine

The intelligence of this machine can be defined from its capability to measure muscle force, provide real-time visual feedback as well as the ability to run in two different modes of exercises. The two different modes in which the machine can run, are namely Continuous Passive Motion (CPM) and Active Resistive Motion (ARM). In CPM mode, the physiotherapist just has to select the CPM mode and key in the number of cycles required. The system is provided with an emergency stop button to protect the patient in case of emergency. In the ARM mode, the force from the foot of the motion measured using the load cells will be compared to a range of set values, within which the machine will keep on moving. If the push force from the feet is lower or higher the system will stop and advise the patient to lower or increase the force from the foot.

\section{RESULTS}

Fig.2 below shows the iLLSRM system prototype being tested with healthy patient. In this setup the patient is requred to push the device and at the same time paying attention to the display on the screen which requires the patient to exert the force in some predetermined level. Fig. 3 shows the real time push force from the healthy leg and the paretic leg which allows for direct comparison of the difference of the push force. This will allow for better communication and also monitoring of patient recovery. 


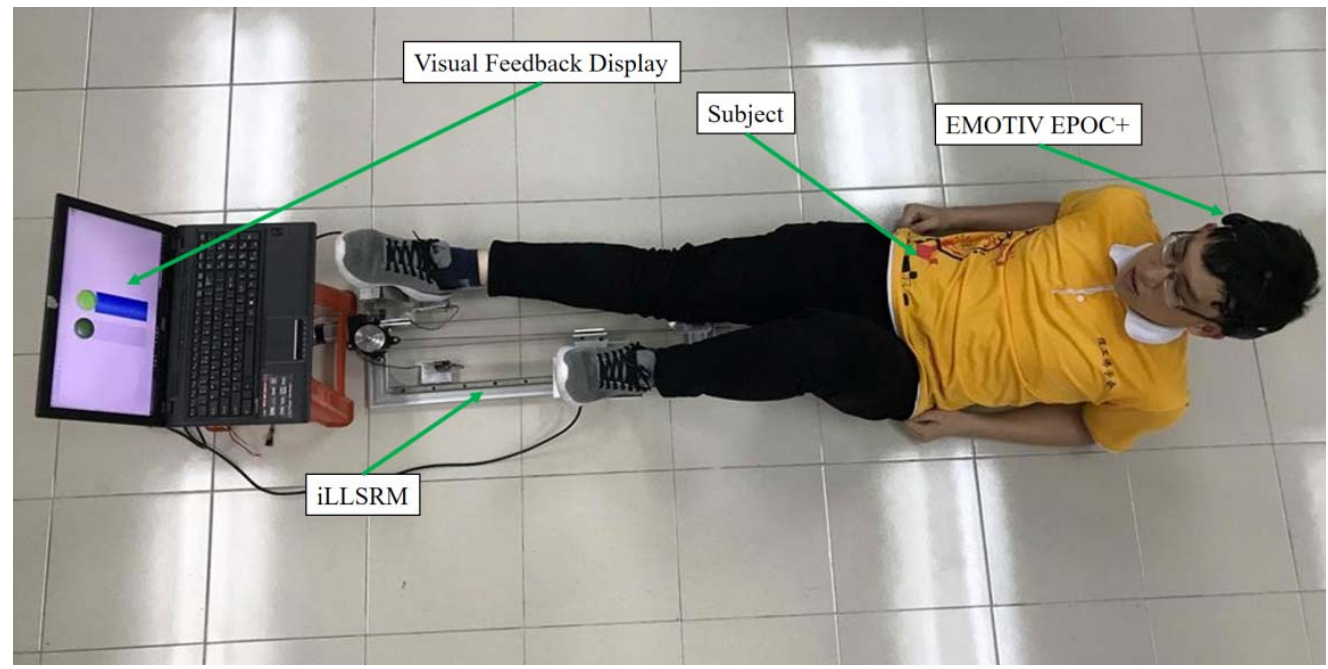

Figure 2. Set-up of iLLSRM System

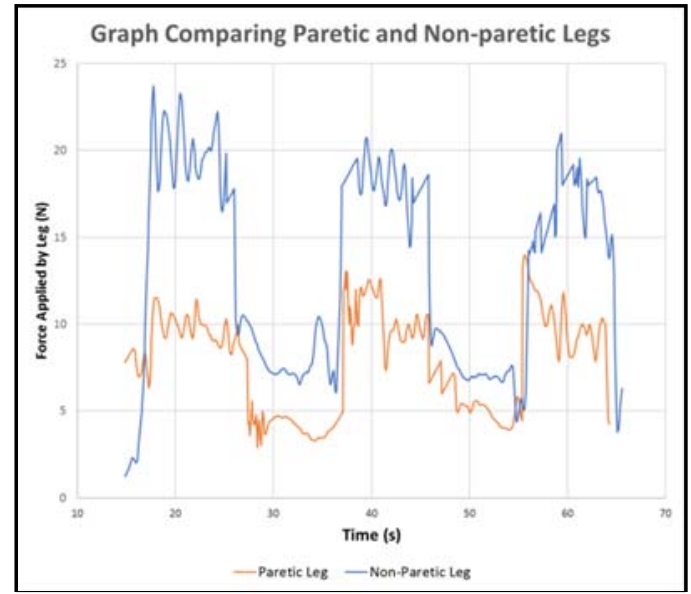

Figure 3. Display Comparing Paretic (stroke affected) and Non-Paretic Leg (nonaffected)

\section{Measuring Engagement}

The results of the engagement measure of all the five healthy subjects with age 21-24 (mean $=22.8 \pm 1.3$ years), undergoing exercises with and without visual feedback is shown in the box plot in Fig. 4 below to compare the engagement measure before and after thefeedback was introduced in the exercise. 


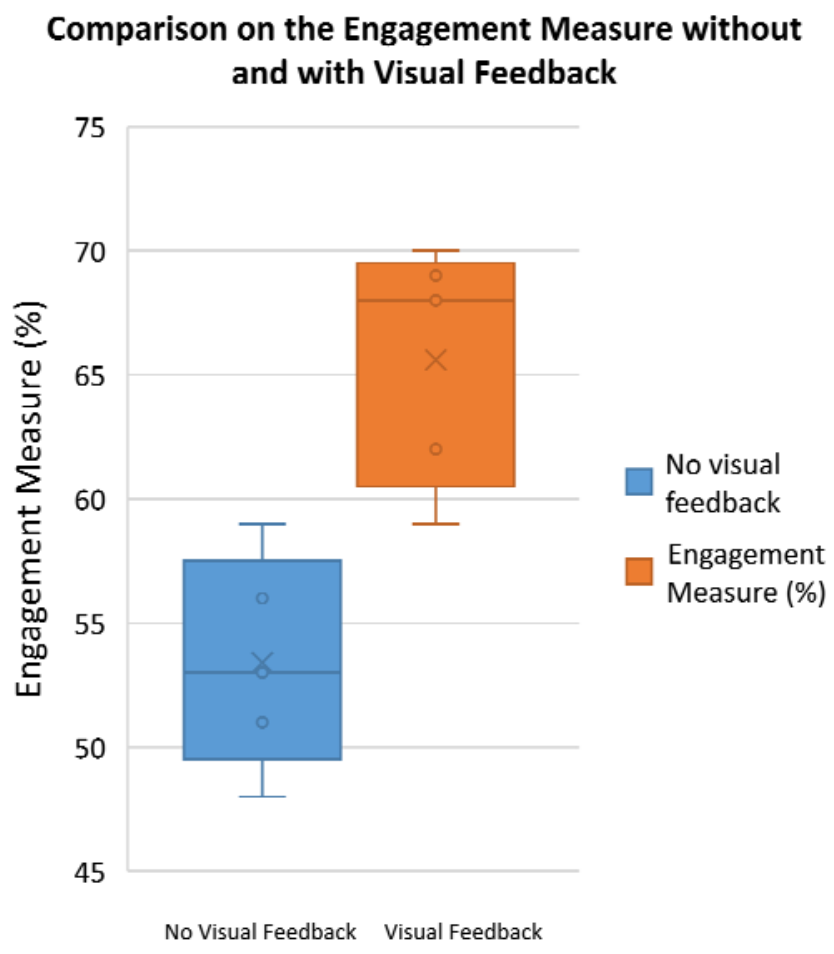

Figure 4. Engagement Measure Comparison for Exercises

\section{DISCUSSION}

The machine has shown its intelligence and capability in monitoring muscle strength recovery, displaying real-time visual feedback and running in two different modes. As shown in Fig. 3, the force exerted by the non-paretic limb is used as a baseline strength to indicate the full recovery of the paretic limb. This is because the strength of the lower limb varies from one person to another. The paretic limb is considered to recover fully once it is able to exert the same magnitude of force as the non-paretic limb. When it is being observed carefully, there is a slight lag off in the graph on force exerted by the paretic limb.

The percentage of increment in engagement measure was $24.53 \%$. Hence, it can be deduced that patients will be more concentrated and highly engaged in their rehabilitation exercises as compared to conventional machines. This is due to the fact that the system interactively communicates to the patients concerning the muscle performance in terms of their range of motion, as well as the minimum force which they have to overcome for the continuous movement. It can maintain the engagement of patients in undergoing the therapy without or with lesser feeling of boredom, as there are certain targets to be achieved in their rehabilitations. At the same time, it will be stimulating the somatosensory cortex in the affected hemisphere, which is known to be involved in cortical reorganization after stroke. 


\section{CONCLUSION}

This project has successfully come out with a lower limb stroke rehabilitation machine which can provide goal-oriented visual feedback in real-time, measuring lower limb muscle force and running in either Continuous Passive Motion (CPM) or Active Resistive Motion (ARM). It is also proven that visual feedback can help patients in being more engaged in their rehabilitation.

\section{ACKNOWLEDGEMENTS}

The authors wish to thank the five healthy subjects, namely D. M. Lau, Y. X. Tan, J. D'raj, K. F. Law and D. Singh, who were involved in the acquisition of restingstate EEG data as well as the experiment of investigating the effect of real-time visual feedback on the engagement. They are all currently the students of Engineering Campus, Universiti Sains Malaysia.

\section{REFERENCES}

[1] Berry, S.M.M.R.V.T., B.R.A.P. Wilson, and L.F.J.M.D.P.M.P.H. Fallon, Strokes. 2017, Salem Press.

[2] Lindsay, P., et al., Toward a more effective approach to stroke: Canadian Best Practice Recommendations for Stroke Care. Canadian Medical Association Journal, 2008. 178(11): p. 1418-1425.

[3] Daniel, K., et al., What Are the Social Consequences of Stroke for Working-Aged Adults? Stroke, 2009. 40(6): p. e431.

[4] Pollock, Alex \& Baer, Gillian \& Campbell, Pauline \& Ling Choo, Pei \& Forster, Anne \& Morris, Jacqui \& Pomeroy, Valerie \& Langhorne, Peter. (2014). Physical rehabilitation approaches for the recovery of function and mobility following stroke. The Cochrane database of systematic reviews. 4. CD001920. 10.1002/14651858.CD001920.pub3.

[5] Jung HY. (2017) Rehabilitation in Subacute and Chronic Stage After Stroke. In: Lee SH. (eds) Stroke Revisited: Diagnosis and Treatment of Ischemic Stroke. Stroke Revisited. Springer, Singapore

[6] Rønning, O.M., \& Guldvog, B. (1998). Outcome of subacute stroke rehabilitation: a randomized controlled trial. Stroke, 29 4, 779-84.

[7] Hebert, D., Lindsay, M. P., McIntyre, A., Kirton, A., Rumney, P. G., Bagg, S., et al. (2016). Canadian Stroke Best Practice Recommendations: Stroke Rehabilitation Practice Guidelines, Update 2015. International Journal of Stroke, 11(4), 459-484. doi: 10.1177/1747493016643553

[8] Poon, C.-S. Sensorimotor learning and information processing by Bayesian internal models. in Engineering in Medicine and Biology Society, 2004. IEMBS'04. 26th Annual International Conference of the IEEE. 2004. IEEE.

[9] Israel, J.F., et al., Metabolic costs and muscle activity patterns during robotic-and therapist-assisted treadmill walking in individuals with incomplete spinal cord injury. Physical therapy, 2006. 86(11): p. 1466-1478.

[10] Wolbrecht, E.T., et al. Real-time computer modeling of weakness following stroke optimizes robotic assistance for movement therapy. in Neural Engineering, 2007. CNE'07. 3rd International IEEE/EMBS Conference on. 2007. IEEE. 
[11] Marchal-Crespo, L. and D.J. Reinkensmeyer, Review of control strategies for robotic movement training after neurologic injury. Journal of neuroengineering and rehabilitation, 2009. 6(1): p. 20.

[12]Kwakkel, G., B.J. Kollen, and H.I. Krebs, Effects of Robot-Assisted Therapy on Upper Limb Recovery After Stroke: A Systematic Review. Neurorehabilitation and Neural Repair, 2007. 22(2): p. 111-121.

[13]Thikey, Heather Anne and van Wijck, Frederike and Grealy, Madeleine and Rowe, Philip (2011) A need for meaningful visual feedback of lower extremity function after stroke. In: 2011 5th International Conference on Pervasive Computing Technologies for Healthcare (PervasiveHealth 2011). Institute of Electrical and Electronics Engineers, pp. 379-383. ISBN 9781612847672

[14]Li, Chong \& Rusák, Zoltán \& Horvath, Imre \& Ji, Linhong. (2016). Validation of the reasoning of an entry-level cyber-physical stroke rehabilitation system equipped with engagement enhancing capabilities. Engineering Applications of Artificial Intelligence. 56. 185-199. 10.1016/j.engappai.2016.09.006.

[15]N. Lehrer and L. Olson (2009). Visual feedback for Mixed Reality stroke rehabilitation, 2009 Virtual Rehabilitation International Conference, Haifa, pp. 194-194.

[16]Physiopedia. Fugl-Meyer Assessment of Motor Recovery after Stroke. Retrieved fromhttps://www.physio-pedia.com/Fugl

Meyer_Assessment_of_Motor_Recovery_after_Stroke 